\title{
Las organizaciones intermedias en los procesos de innovación en México
}

\author{
Maria del Pilar Monserrat Pérez Hernández*
}

Perfiles Latinoamericanos, 24(48)

2016 | pp. 161-183

DOI: $10.18504 / \mathrm{pl} 2448-007-2016$

\begin{abstract}
Resumen
La actividad innovadora depende de los vínculos o mediación que exista entre los actores que generan la tecnología y el conocimiento y los usuarios finales de una y otro; este papel lo cumplen diversas organizaciones intermedias (OI), las cuales traducen las necesidades de los usuarios de tecnologías en desarrollo. Este trabajo analiza el papel de dichas organizaciones intermedias en los procesos de innovación en México. Así, se distingue que existen tres vacíos estructurales: en la negociación y el patrocinio, en la comercialización y explotación de los resultados, y en la regulación y arbitraje, mismos que inhiben la apropiación de los beneficios derivados de la actividad innovadora.
\end{abstract}

\begin{abstract}
The innovation activity depends strongly on the interaction between generating actors and end-users of new knowledge and technologies. There are several intermediate organizations (IO) that facilitate translating the needs of the users with the new technologies that are being developed, in order to satisfy more effectively their demands. This paper analyzes the mediation process involved in the IO in innovative activity in Mexico, to identify structural holes in the innovation process. The relevant results in the Mexican intermediation process points that three important structural holes of the process are missing: one between the negotiation process and sponsorship, the second in marketing and commercial profit of the obtained results and the last one in the regulation and referring activities. It is very important that the detected missing links in the intermediation process are filled, because they can hinder considerably the overall profit and benefits that society could obtained of the innovation process.
\end{abstract}

Palabras clave: intermediación, organizaciones intermedias, innovación, México, vacíos estructurales. Keywords: Brokerage, intermediary organizations, innovation, Mexico, structural holes.

* Doctora en Economía y Gestión de la Innovación y Política Tecnológica por la Universidad Autónoma de Madrid (Espańa). Profesora del Instituto Politécnico Nacional (México). 


\section{Introducción}

- os profundos cambios en la economía mundial originados por la globalización, la regionalización, la liberalización económica y la revolución tecnológica, provocaron lo que Freeman (1987) denominó la evolución y gradual aparición de nuevos tipos de instituciones y mecanismos para el desarrollo de innovaciones en países y regiones.

La actividad innovadora son todas las tareas científicas, tecnológicas, organizativas, financieras y comerciales, incluyendo la inversión en nuevo conocimiento, que conducen real o potencialmente a la puesta en marcha de innovaciones. La naturaleza de la actividad innovadora varía ampliamente de una empresa a otra (OCDE, 2005).

La decisión de innovar se toma en situaciones con un alto grado de riesgo e incertidumbre (Rosenberg, 1994). Los desarrollos futuros de conocimiento y tecnología, los mercados, la demanda de un producto y los usos potenciales de una tecnología son impredecibles y pueden variar en función del sector, del ciclo de vida del producto, de la adopción de nuevos productos o procesos, así como de la puesta en marcha de nuevos métodos comerciales u organizativos (OCDE, 2005).

Debido a la complejidad de la actividad innovadora han cobrado relevancia las organizaciones intermedias $(\mathrm{OI})$, mismas que desempeñan muy variadas tareas en el proceso de innovación.

Las OI son agentes, enlaces o brokers que facilitan alguna actividad innovadora entre dos o más partes. Con su experiencia y capacidades buscan crear un ambiente de confianza y certidumbre que coadyuve al aprendizaje, la creatividad y el trabajo en red. Es decir, sirven de conexión o puente entre los generadores de tecnología y los usuarios de la misma, para lo cual asumen una diversidad de funciones con el propósito de introducir al mercado nuevos productos, procesos y métodos organizacionales o comerciales.

A pesar de la diversidad de dichas organizaciones, lo común a todas es que su proliferación contribuye a fortalecer el contexto institucional, pues crean mecanismos de mediación y colaboración entre los actores de la actividad innovadora.

El objetivo de este trabajo es analizar el proceso de mediación en el que intervienen las OI en la actividad innovadora en México, para identificar los vacíos estructurales en el proceso de innovación. La metodología ha consistido en una revisión de la literatura más la aplicación de una encuesta a los directivos de las OI seleccionadas. Este artículo consta de cuatro secciones: la primera es esta introducción; la segunda, analiza la naturaleza y el papel de las OI, incluyendo su evolución en la actividad de innovación, y la importancia que en esta tiene la mediación. En la tercera, se presentan la naturaleza y evolución de las OI en 
México, donde se identifican algunas peculiaridades de los países emergentes, además de los vacíos estructurales en la actividad de innovación en México. La cuarta parte se dedica a las reflexiones finales.

\section{Naturaleza de las organizaciones intermedias}

Las OI han sido poco analizadas y los estudios al respecto son empíricos y teóricamente fragmentados, enfocados en el análisis de casos. Incluso la literatura de los sistemas nacionales de innovación concibe las OI como firmas, desempeñando cada una un rol o función específica dentro del sistema (Jaso, 2011). El interés en su papel en el proceso de innovación ha emergido, como señalan Howells (2006) y Van der Meulen (2007), de diferentes fuentes y campos de investigación.

Las diversas acepciones de las OI han variado tanto en su denominación y funciones, como en su papel en la actividad innovadora. El significado genérico de "intermediario" es cualquier organización que participe en la relación entre dos o más actores sociales. Por ello, en este trabajo se entenderá por OI las entidades que actúan como interfaz o brokers para facilitar alguna actividad innovadora entre dos o más partes, experiencia con la cual buscan generar un ambiente de confianza y certidumbre que coadyuve al aprendizaje y trabajo en red. Es decir, coadyuvan a que los usuarios y generadores de tecnología cooperen para la exitosa introducción de innovaciones.

La heterogeneidad de las funciones de las OI, su flexibilidad organizativa y su escasa burocratización les han permitido construir un mercado integrado de servicios, debido a que vinculan activamente a las empresas, los centros de investigación y el sector público orientado al fomento productivo (Casalet $\&$ González, 2006).

Las OI también se distinguen por su carácter híbrido, y su aparición y consolidación es una expresión clara del modelo interactivo de innovación que subraya la creación de conocimiento en distintas esferas institucionales, redes y organizaciones híbridas de investigación (Lam, 2002). Asimismo, hay que considerar que las OI pueden cambiar de actividades y roles (Jaso, 2011).

Autores como Van Lente et al. (2005), Van der Meulen (2007) y Stezano (2009) consideran que las razones para el crecimiento, expansión y diversidad de las OI son:

a) La naturaleza del conocimiento científico y la investigación y el lugar que tales organizaciones ocupan en la vida social y en el debate político, además de su inclusión en las políticas públicas. 
b) La mayor complejidad de conocimientos e innovaciones se produce y sostiene por redes de empresas e instituciones, lo cual da un carácter más distribuido a los sistemas de innovación.

c) La imitación trasnacional y ciertas modas en las políticas públicas. Entorno que requiere de un trabajo de colaboración entre múltiples instituciones, funciones y fronteras del que surgen actividades de intermediación.

d) El origen del financiamiento gubernamental a la ciencia e investigación y cuestiones vinculadas con su autonomía e independencia.

e) El cambio de lugar e importancia de la ciencia y la aparición de redes ciencia-industria, para crear diferentes tipos de organizaciones y procesos de participación, sobre todo en la traducción de conocimientos y la capacidad para agregar intereses de actores heterogéneos.

f) La existencia de distintos ámbitos de mediación y formas organizacionales.

\section{Evolución de las organizaciones intermedias en la actividad innovadora}

La evolución histórica de los intermediarios en ciencia, tecnología e innovación (СтI) está asociada a temas como el financiamiento público, la preocupación por la autonomía e independencia de su quehacer, la percepción de la academia, y la necesidad de incorporar el conocimiento generado por las instituciones de educación superior (IEs) con el sector productivo (Van der Meulen, 2007).

En el caso de Estados Unidos, desde la década de 1940 el financiamiento público otorgado a la ciencia permitió la proliferación de las OI (Baxter \& Tyler, 2007). Para los sesenta, la disminución de esos fondos públicos forzó la búsqueda de otras fuentes de financiamiento, lo que produjo los primeros esfuerzos de transferencia del conocimiento hacia las empresas, unas veces con éxito y otras con desencuentros y lecciones aprendidas. Es hasta 1980, con la aprobación de la ley Bayh-Dole de Estados Unidos, ${ }^{1}$ que se establece el marco institucional para que una serie de OI consolide su colaboración con los agentes de la actividad innovadora (Varela, 1997; Stezano, 2009). Incluso las propias IEs crearon mecanismos dentro y fuera de ellas que fomentaban la comercialización de la tecnología que generaban.

En la actualidad, en muchos países las relaciones entre academia, gobierno e industria las median organizaciones que se dedican a ello. Los patrones de mediación y las formas organizacionales varían de manera sustancial entre los

1 La cual autoriza a las universidades a cobrar regalías por las invenciones patentables que resulten de proyectos de investigación financiados por el gobierno federal. 
diferentes ambientes nacionales, pero históricamente ha habido una tendencia general hacia la expansión de los intermediarios. El proceso de mediación se debe a factores que se extienden desde la naturaleza del conocimiento científico y tecnológico y el lugar que este ocupa en la vida social y el debate político, hasta la imitación transnacional y política y las modas estructurales (Van der Meulen, 2007).

Por ello, las OI han asumido diferentes denominaciones, características y funciones que denotan su dinámica y constante evolución; sin embargo, un elemento común entre ellas es el proceso de intermediación que realizan. En la figura 1 se observan las modalidades de OI según la esfera que les da vida (lugar, conocimiento, financiamiento y política). A pesar de su diversidad, estos mecanismos comparten el arraigo y la especificidad local.

Figura 1. Taxonomía de organizaciones intermedias presentes en la actividad innovadora

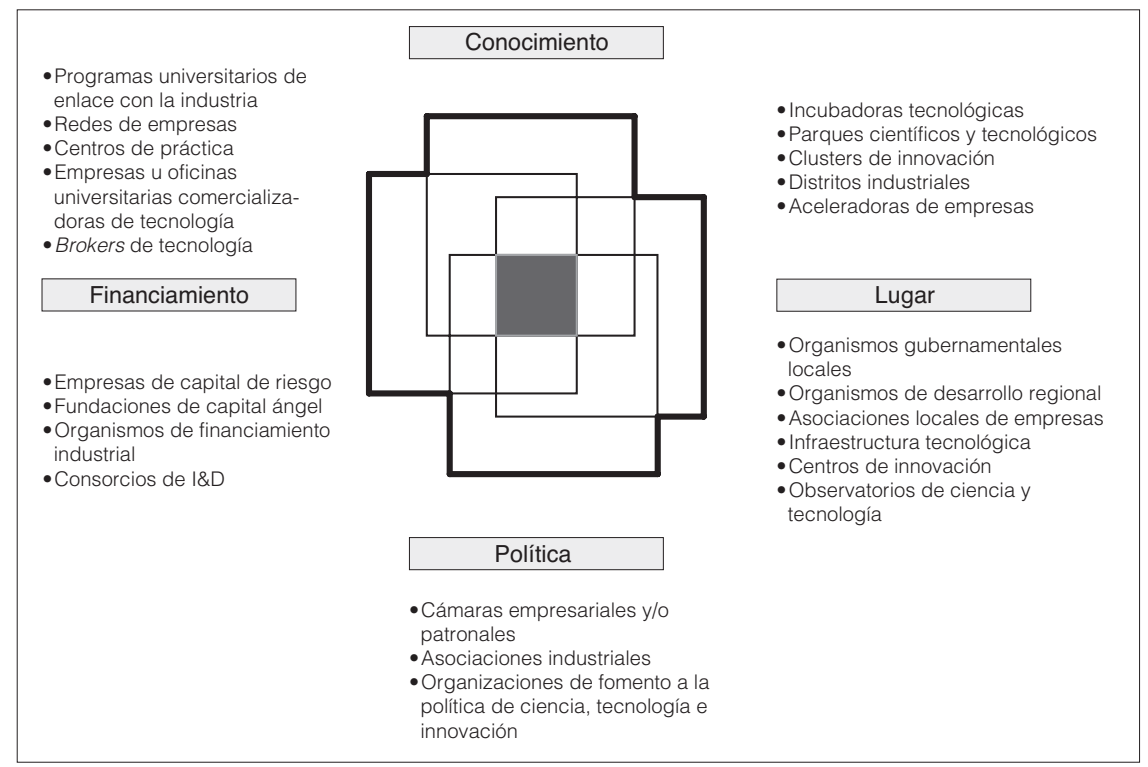

Fuente: Pérez (2014).

El papel de las organizaciones intermedias en el proceso de innovación

El papel que las OI han desempeñado en la conformación de los sistemas de innovación permite analizar sus rasgos sistémicos y sinérgicos, pues, como se ha visto, articulan, median y coadyuvan en el proceso de aprendizaje de los actores, 
pero al mismo tiempo solventan fallas, problemas o debilidades del sistema de innovación (Szogs, Cummings \& Chaminade, 2009).

La teoría evolucionista del cambio tecnológico se refiere a las OI — cuando se ocupa de la actividad innovadora - como sistémicas y necesarias para desarrollar interacciones entre empresas, territorios y países, y destaca la influencia que ejerce la estructura de producción y el marco institucional (Edquist \& Johnson, 1997; Lundvall et al., 2002).

Por lo anterior, es relevante distinguir si los intermediarios son sujetos o parte del proceso de mediación. Esta discusión es pertinente, pues la diversidad y heterogeneidad de las OI indican que más allá de la forma, estas contribuyen en distintos segmentos de la actividad innovadora.

La literatura refiere la intermediación como una función particular; sin embargo, al analizar los estudios de actores intermediarios se les reconoce un papel más amplio, dinámico, diverso y holístico en el proceso de innovación (Howells, 2006; Casalet, 2012; Jaso, 2011). En general, se han analizado casos de estudio de la trayectoria de las OI, entre los que se pueden mencionar a Jaso (2011) y Ekboir et al. (2009), o el papel que cumplen en un sector o industria, por ejemplo, en software, Casalet et al. (2008) y Rodríguez (2011), y, en biotecnología, Pérez (2011) y Stezano (2012).

Van der Meulen (2007) y Casalet et al. (2008) hablan del liderazgo que asumen los intermediarios en un determinado sistema o industria a través de programas y estrategias de innovación. En industrias específicas, las OI cuentan con conocimientos más completos acerca de las tecnologías clave que operan, o bien poseen un capital relacional que les permite proveer y difundir conocimiento acerca de la tecnología.

El papel de las OI se alude con las metáforas de la "polinización cruzada" o "tendedor de puentes" entre grupos desconectados o inconexos, por ejemplo, vinculan miembros de un grupo social en particular con nuevas ideas creadas o inventadas dondequiera (Howells, 2006). Sin embargo, esa misma diversidad hace necesario ubicarlos en un marco analítico donde se desempeñan con ese fin, apuntando esto a la noción de mecanismo regulador de las dimensiones productivas e institucionales de las regiones.

La capacidad de las OI depende de tres factores: $a$ ) tipo de relación entre los actores (traslape de intereses y la asimetría en la posición de recursos), b) posición de las OI en los recursos, y c) institucionalización de la relación y posición del intermediario (Van der Meulen, 2007).

La función que desempeñan las OI se vincula a la creación de un entorno de confianza y certidumbre, pues originan redes de colaboración entre diferentes actores, lo que facilita la interacción y el aprendizaje. Asimismo, la posición estructural de las OI puede cambiar, debido a que está permeada por el contexto 
político (en raras ocasiones se desempeña como un actor imparcial). Al estar asociadas a los procesos de creación y difusión de las innovaciones y del conocimiento, así como a las modificaciones y la recomposición de los mercados, las OI se encuentran en constante evolución (Nelson, 1998; Van der Meulen, 2007; Vázquez, 2006).

En general, en la literatura se aborda poco a los actores que median entre los creadores de invenciones y los usuarios. Cuando surge una innovación, el sendero de difusión sufre transformaciones; el ambiente institucional en el cual tiene lugar la difusión también se modifica en respuesta a los efectos esperados o realizados de la adopción extendida de la innovación. Varias de las OI forman parte de este ambiente. Los modelos tradicionales de difusión por lo común muestran que su paradigma supone una dinámica de interacción entre dos partes: los generadores de nuevos productos y procesos y los potenciales adoptadores de tales innovaciones (Mantel \& Rosegger, 1987).

Los diversos modelos analíticos de las OI no reconocen que estas se transforman en el proceso de difusión. Es más frecuente que la acumulación de información sea acorde con el papel clave que desempeñan estas organizaciones que no solo brindan servicios especializados "de una vez y para siempre" a sus clientes, sino que también los asisten con capacidades innovadoras relacionales de largo plazo (Mantel \& Rosegger, 1987).

Se distinguen dos tipos de enfoques analíticos de los intermediarios: a) como organizaciones y $b$ ) la intermediación como proceso. En esta investigación se optó por el segundo enfoque, en el cual se retoman las diferentes actividades de intermediación.

Mediación de las OI: elemento clave de la actividad innovadora

El factor organizacional de la intermediación es el elemento central, las OI cumplen diversas funciones medulares para los sistemas de innovación, su importancia radica en que explican con efectividad la importancia de su función de compensar debilidades de los sistemas de innovación.

La mediación implica la existencia de dos actores de distinta naturaleza y base de conocimiento, por lo que se requiere una traducción de significados, resultados o intereses de uno de ellos, o de ambos. La coordinación institucional resalta qué tan crítica es la coordinación en la actividad innovadora (Van der Meulen, 2007).

Howells (2006) resalta cinco elementos clave de la intermediación:

1. Es más numerosa y diversa de lo que los estudios hayan indicado. A lo largo de la cadena de valor, los intermediarios proveen funciones como: $a$ ) movimientos 
contracorriente (scoping e inteligencia) o corriente abajo (protección y comercialización de la propiedad intelectual), b) diversidad dentro de nuevas industrias y tecnologías, y c) cambios hacia nuevos mercados extranjeros.

2. Interviene en relaciones cada vez más complejas. En un contexto de múltiples vínculos de redes de intermediarios, estos adquieren más importancia y también transforman otros atributos, como el poder de dependencia entre intermediarios y clientes.

3. Ayuda a sus clientes a desarrollar innovaciones. Empero, de modo creciente, los clientes se vuelven más proactivos en esta etapa.

4. La relación con sus clientes es de corto y largo plazo y vincula cada vez más las capacidades de innovación colaborativa. Esas colaboraciones pueden ser de años y proveen oportunidades para que los intermediarios brinden servicios de mayor valor agregado.

5. Diferenciación entre las actividades de intermediación en la innovación, lo que puede incluir una amplia gama de funciones.

Así, la coordinación se coloca como un elemento crítico de la intermediación; la mediación corresponde a la coordinación, delegación de autoridad o gobernanza, en donde la negociación o brokering son mecanismos que traspasan las distancias entre grupos inconexos, al facilitar información, liderar la gestión, y controlar proyectos conjuntos y la coordinación de sus actividades (Vázquez, 2002; Stezano, 2009).

Ese trasfondo institucional es central para la coordinación de los procesos de innovación; el papel de las OI contribuye a reducir los costos de transacción, aporta mayor confianza a la interacción y se expresan sobre demandas reales (Vázquez, 2002; Stezano, 2009).

Otro tema asociado es la cooperación que permite la difusión del conocimiento y el aprendizaje. El conocimiento tácito fluye con dificultad en relación con el conocimiento explícito, pues el tácito se encuentra incorporado en la gente, la cultura y la estructura organizacional, y solo puede ser sustraído parcialmente (Nooteboom, 2000; García, Lara \& Taboada, 2004).

Finalmente, hay que considerar que el desarrollo de las OI es circunstancial con el contexto político y que en pocas ocasiones es un actor imparcial. El ámbito donde se desempeñan está influido por el surgimiento de nuevas funciones o coyunturas en los diferentes sistemas de innovación y producción, por el nacimiento de nuevas OI que compiten en similares actividades con las ya existentes, y por la competencia entre ellas por satisfacer o convertirse en interlocutores de los nuevos espacios que se generan en el marco institucional. Las OI son flexibles, pues aprenden a relacionarse y conocer, ya que actúan en contextos cambiantes. 
Por ello, colocar las OI en la actividad innovadora como objeto de investigación pone de relieve que, para que una región o localidad sea innovadora o exitosa, no basta tener infraestructura y recursos, es menester un entramado institucional que facilite los procesos de interacción, como son el aprendizaje, la transferencia de tecnología o la gestión tecnológica, etcétera. Ese entramado institucional soporta el conocimiento tácito y el aprendizaje que se generan en la región, el cual es influido por la cultura y las formas de gobernación y repercute en el funcionamiento de las redes y las formas de cooperación de los agentes que participan en la actividad innovadora (Pérez, 2014).

Existen tres propuestas que consideran, desde una perspectiva sistémica, la actividad de intermediación: la taxonomía de Howells (2006); la tradicional — enfocada al destino y uso del conocimiento - y la que analiza funciones, propuestas por Galli \& Teubal (1997). En la tabla 1 se presentan las tres taxonomías y se agrupan conforme al tipo de conocimiento que asocian.

Tabla 1. Taxonomías y funciones de intermediación de las organizaciones intermedias

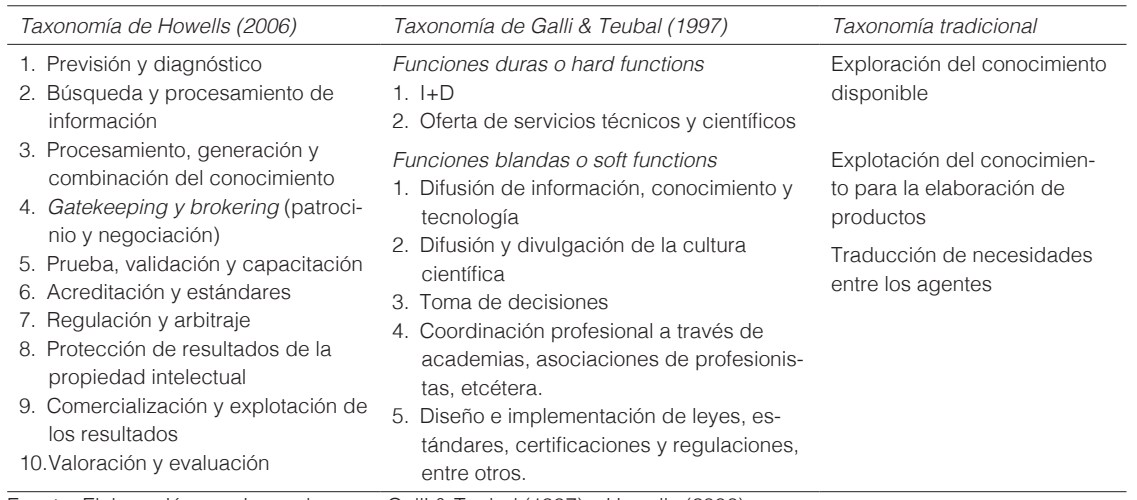

Fuente: Elaboración propia con base en Galli \& Teubal (1997) y Howells (2006).

Uno de los propósitos de este trabajo es la identificación de vacíos estructurales en el proceso de innovación a partir del mapeo de las funciones de intermediación requeridas para realizar la actividad innovadora, para lo cual se utilizó la categoría de vacíos en el proceso de innovación, de acuerdo con Stezano (2009), que retoma la teoría de los hoyos estructurales del capital social propuesta por Burt (2002). Un vacío estructural entre dos grupos implica que las personas de cada uno de ellos están concentradas en sus propias actividades, de forma que no pueden atender las actividades del otro grupo. Los vacíos son una interfaz o una oportunidad para traspasar, pues las personas en cada lado circulan con diferentes flujos de información, por lo que el intermediario 
se convierte en la interfaz que trae información y conocimiento de otras personas desde lados opuestos del vacío (Burt, 2002).

Dada la desconexión existente entre dos o más grupos es de esperar que desarrollen sus propios lenguajes, creencias y prácticas de negocios. Lo que es una ventaja potencial para la integración de redes que tiendan puentes entre esos grupos y coordinen sus actividades. Las redes con múltiples vacíos estructurales presentan oportunidades para un comportamiento emprendedor (Stezano, 2009).

En el enfoque de los vacíos estructurales, una OI puede cambiar su posición en la red, pues es capaz de gestionar el control de recursos, desarrollar su propia función o colocar a otro actor que cubra esas necesidades.

Van der Meulen (2007) afirma que las OI son competentes para asumir funciones de liderazgo decisivo en un determinado sistema, como puede ser la ejecución de programas políticos concretos, por ejemplo, estrategias de innovación a escala nacional, en donde los intermediarios desempeñan una multiplicidad de papeles, desde el broker o tendedor de puentes entre agentes - al proveer información y conocimiento-, hasta tareas más vinculadas con el diseño de políticas públicas.

Estos enfoques son de gran utilidad para comprender el desarrollo de las OI en países emergentes como México. Con el propósito de identificar en dónde se encuentran los vacíos estructurales en la actividad de intermediación se propone que, a partir del modelo de ciclo de vida de la innovación, planteado por Abernathy \& Utterback (1978) y Tidd \& Bessant (2009), se incorporan las fases del proceso de innovación propuestas por Whitman (1989) y Pearson (1990). En la figura 2 se muestran las etapas y el ciclo de la innovación.

El proceso de innovación, de acuerdo con Pérez (2011), consta de cuatro etapas:

a) Emergencia: a partir de la identificación de oportunidades tecnológicas o la atención de necesidades se desarrolla una tecnología surgida de la invención, llamada tecnología emergente. El impacto potencial es desconocido pero prometedor, pues se muestra ya su potencial. La tecnología está en las primeras etapas de su aplicación, que comprende la identificación de la oportunidad, formulación de la idea, solución del problema y el desarrollo del prototipo.

b) Crecimiento: a lo largo de esta fase se da una intensa mejora de la tecnología, hasta ser lo suficientemente fiable como para materializarse en algunas aplicaciones importantes. Se busca una utilización más económica, donde se pueden cambiar componentes de la tecnología parar resolver problemas, aumentando su rendimiento y encontrando campos de aplicación y nuevas funciones. Asimismo, se desarrollan tecnologías claves.

c) Madurez: la experiencia adquirida por la tecnología en aquellos campos en los que se ha implantado y desarrollado, permite resolver los principales problemas 
de utilización, estabilizándose sus procedimientos operativos, así como sus rendimientos. Se da un desarrollo considerable de los campos de aplicación de la tecnología, pues al ser bien conocida se pueden explotar todas sus posibilidades de uso. Uno de los objetivos es buscar el desarrollo extensivo de la tecnología; en esta fase se da a conocer al público en general.

d) Saturación: la tecnología alcanza su límite tecno económico o de productividad y rendimiento, o bien alcanza la auto saturación por la incapacidad que tiene la tecnología para responder a las crecientes exigencias, lo que da paso al surgimiento de otra tecnología.

Figura 2. Ciclo de vida de la tecnología y las etapas del proceso de innovación

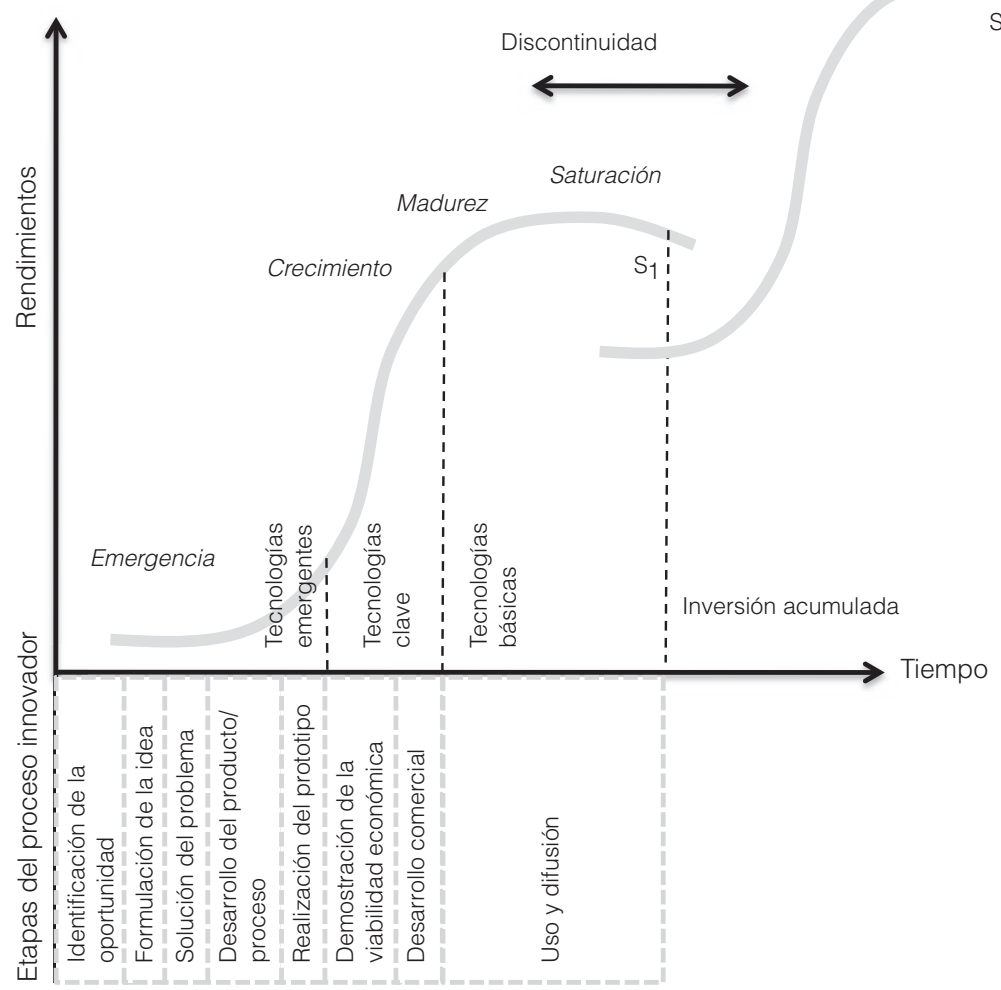

Fuente: Elaboración propia con base en Abernathy \& Utterback (1978), Tidd \& Bessant (2009), Whitman (1989) y Pearson (1990).

Respecto al desempeño de las OI en el proceso innovador y en el ciclo de vida de la innovación, en la figura 3 se correlacionan la ubicación de las activi- 
dades de intermediación con las etapas del proceso de innovación. La combinación de ambos elementos busca identificar los vacíos estructurales que dificultan el ciclo de las innovaciones.

Figura 3. Relación entre el proceso de innovación, el ciclo de vida de la tecnología y las funciones de intermediación

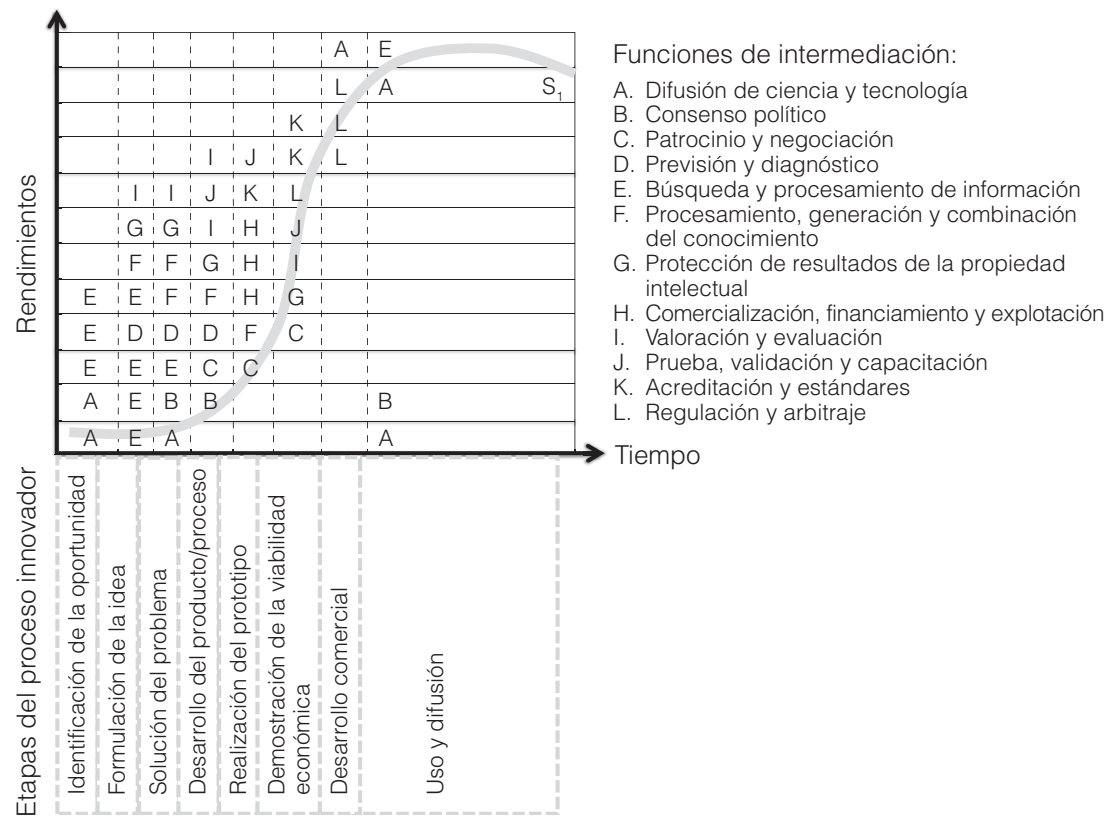

Fuente: Pérez (2011).

De esta forma, al considerar las diferentes funciones de intermediación que realizan las OI, con el ciclo de vida de la innovación se pueden identificar los vacíos estructurales en el proceso de innovación. La presencia de aquellos dependerá de cada estructura industrial y de servicios. A continuación se presenta el caso mexicano.

\section{Naturaleza de las organizaciones intermedias en la actividad innovadora en México}

El papel clave de las OI es que solventan fallos, problemas o debilidades del sistema de innovación. Por ello en esta sección se abordarán las peculiaridades 
de las OI en países emergentes, para luego analizar su evolución en México, y cerrar con la identificación de los vacíos estructurales de la actividad innovadora en este país.

\section{Peculiaridades de los países emergentes}

Las OI desempeñan diferentes roles de acuerdo con el grado de desarrollo de los países. Así, en países industrializados con una estructura institucional madura, los intermediarios tienen funciones definidas que dan pie a una constelación de OI que atienden los requerimientos de la actividad innovadora. En cambio, en países emergentes, el marco institucional es "poroso" y las OI se orientan a más de una actividad; su adaptación al cambio depende del sector tecnológico o social en el que surgen los esfuerzos por conformarlas. Las OI regionales aún están en proceso de construcción y predominan las de nivel nacional (Doner, Hicken \& Ritchie, 2009; Szogs, Cummings \& Chaminade, 2009).

En los países emergentes, la fragilidad del marco institucional ante los cambios en el contexto tecnoeconómico permite la emergencia de nuevas instituciones, y la declinación y desaparición de las anteriores. Las que sobreviven será porque fueron más racionales en cuanto a su organización y más eficiente en el uso de sus recursos y de las fuentes tecnológicas de la actividad productiva (Casalet, 2000).

Aunque, como afirma Casalet (2000), la existencia de grupos de poder y coaliciones a través del gobierno dificultan los cambios, pues ellos siguen sus propias agendas. La evidencia indica que la distribución basada en el poder político produce mecanismos adversos que condicionan los objetivos de cada organización.

\section{Evolución histórica de las OI en México}

En México, Cimoli (2000) y Dutrenit et al. (2006) sostienen que, en un sistema de innovación en proceso de consolidación, la existencia de las OI compensa las debilidades del sistema.

Por su trayectoria histórica, México presenta un importante capital institucional derivado del corporativismo, un recurso que puede ser determinante para asegurar que las empresas accedan a recursos de información y conocimiento que les permitan introducir innovaciones y ser competitivas. El corporativismo, debido a su estructura jerárquica y a su alta dependencia respecto del gobierno, conviene para acuerdos nacionales de política macroeconómica más que para arreglos meso y microeconómicos. 
Las OI complementan el papel desempeñado por las instituciones especializadas de fomento productivo. Emergen con la apertura comercial y el cambio estructural de mediados de los ochenta (Casalet, 2004).

En los noventa, las OI adoptaron estructuras organizacionales cada vez más flexibles y se adaptaron a las restricciones presupuestarias y de subsidios, fortaleciendo el mercado de servicios, es decir, canalizaron en aquel momento una dinámica de interacción y conocimiento con creciente importancia local e industrial. Esto se sumaba a una estrategia financiera, pues su supervivencia dependía de la habilidad para desarrollar sus propias estructuras por medio de mayor transparencia en sus operaciones y ofreciendo una alta calidad en los servicios (Casalet, 2000, 2004).

Las asociaciones empresariales mexicanas priorizaron los asuntos políticos y relegaron el desempeño económico de las empresas a un segundo plano. ${ }^{2} \mathrm{La}$ tradición corporativista generó un grupo de OI cupulares bien integrado y hábil para negociar con el gobierno, entre las que se pueden mencionar la Cámara Nacional de la Industria de la Transformación (Canacintra), la Cámara Nacional de Comercio, el Consejo Coordinador Empresarial y la Confederación Patronal de México (Coparmex), entre otras.

El marco económico y de competitividad presionó a las empresas para conseguir estructuras más flexibles, competitivas y eficientes, lo que supuso que demandaran servicios para el control de calidad, normalización, certificación de procesos y modernización tecnológica. Tales requerimientos crearon un mercado de servicios especializados de apoyo a la innovación. Así, las OI dedicaron esfuerzos para desarrollar nuevas competencias y enfrentar las demandas de las empresas que, mediante la interacción entre los agentes, facilitara la creación y transferencia de conocimiento (Casalet, 2000).

El marco institucional de la política en ciencia y tecnología de fines de los noventa produjo una serie de mecanismos de intermediación como la Conferencia Nacional de Ciencia y Tecnología, la Red Nacional de Consejos y Organismos Estatales de Ciencia y Tecnología (Renacecyt), los Fondos Mixtos y Sectoriales. Todos ellos con el fin de propiciar y consolidar el aumento de capacidad de gestión, negociación e innovación de los grupos sociales, así como una estrategia de participación estatal para fomentar propuestas regionales (Casalet, 2008).

Más recientemente, se impulsaron instancias como el Comité Intersectorial de Innovación, el Fondo Sectorial de Innovación, los programas públicos para el fomento y consolidación de oficinas de transferencia de conocimiento (OTC), la Red Mexicana de Oficinas de Transferencia de Tecnología, la proliferación

2 Más aún, el ingreso garantizado de las membresías ocasionado por la legislación, provocó que fuera relativamente innecesario ofrecer servicios importantes y de calidad (Tirado \& Luna, 2001). 
de parques tecnológicos en diversas entidades del país, el Sistema Nacional de Incubadoras de Empresas y las Aceleradoras de Empresas (Pérez-Hernández \& Calderón-Martínez, 2014).

En el caso mexicano, el surgimiento de las OI ha respondido a diversos factores, entre los que se pueden mencionar:

a) La búsqueda de interlocutores en países receptores de tecnología. El surgimiento de OI que, con patrocinio de las empresas trasnacionales y gobiernos con acuerdos de comercio, se han transformado al pasar de ocuparse de funciones de difusión a ser interlocutores políticos.

b) Organizaciones de grupos de profesionistas y funcionarios vinculados a la CтI que, unidos por las inquietudes de fomentar la innovación, se han establecido formalmente y han acumulado capital relacional para participar activamente en el diseño de políticas e instrumentos de fomento a la innovación.

c) Organizaciones derivadas de las necesidades de modernización y apertura comercial.

d) Iniciativas académicas y empresariales para adecuarse a las actividades de vinculación con las empresas.

Para analizar el desarrollo de las OI en México se debe diferenciar el tipo de organización. Existen aquellas relativamente recientes, no solo en términos de tiempo sino también en cuanto al entramado institucional o madurez de sus relaciones y vínculos, así como otras que han evolucionado y consolidado su posición en tanto actores clave en la actividad innovadora. Los escasos estudios que existen de OI se enfocan en este último grupo, esos son los casos de ADIAT (Jaso, 2011), Cofupro (Ekboir et al., 2009) y Fumec (Isordia et al., 2011).

La identificación de las OI en el sistema mexicano de innovación es compleja por diversas razones. La principal es que su papel de intermediación lo realizan a través de una amplia variedad de actividades. Pero también porque operan desde diferentes niveles legales y muchas surgieron en los noventa, lo cual implica que aún pasan por las etapas de gestación y consolidación de sus servicios. Asimismo, la evaluación de su desempeño es difícil debido a su propio rol de intermediación, que no es sencillo de cuantificar (Casalet, 2000); por ello, el uso de herramientas cualitativas se convierte en un tema central.

La aparición de nuevas OI, así como del interés por las actividades de investigación y desarrollo, se refleja en la novedosa oferta de mejores servicios y en que las OI compiten con otras asociaciones para atraer afiliados voluntarios más participativos y exigentes. La competitividad ha forzado a las empresas a invertir crecientemente en intangibles. Sin embargo, la sola creación de estas instituciones 
no fue suficiente para asegurar que la red se vinculara con el sistema productivo. Así, ese interés por las actividades de investigación y desarrollo varía de una asociación a otra, algunas han empezado a actuar en estos problemas, mientras que otras permanecen cerradas (Tirado \& Luna, 2001; Casalet, 2000).

A diferencia de Isordia et al. (2011), que solo analiza dos funciones de las propuestas por Howells (2006), este trabajo se ocupa del conjunto de las actividades que este último menciona más otras dos que propone Pérez (2014) de acuerdo a las características de México.

Identificación de vacios estructurales en la actividad de innovación en México

Para determinar la existencia de vacíos estructurales que obstaculicen la actividad innovadora en México en el ámbito de intervención de las OI, aquí se han incorporado dos funciones a las diez propuestas por Howells: a) la difusión y divulgación de la ciencia y tecnología, y b) la actividad de consenso político o lobbying. Estas funciones emanan de la propia naturaleza del contexto mexicano.

Además, se retoman los resultados de la encuesta de Pérez (2011) sobre las OI a nivel nacional. Esa encuesta constó de tres secciones: a) los datos básicos, b) la exploración de los datos de la organización (estatus legal, tamaño de la OI, fuentes de financiamiento, principales clientes y socios), y c) se preguntó acerca de las doce funciones de internación (figura 3 ) y las actividades que se les asocian.

Con el propósito de analizar la intensidad de las actividades citadas, en cada función la encuesta interrogaba sobre el grado de formalización (la existencia o no de contratos o convenios); la frecuencia (con cuatro opciones: nunca, casi nunca, una vez al año y más de dos veces al año) y con quiénes se efectuaban las actividades (ámbito nacional o local y si eran empresas, academia o gobierno). Si la OI la cumplía rutinariamente eso mostraba su capacidad para atender la necesidad y el éxito en su desempeño. Todo lo anterior permitió conocer si la actividad era cotidiana, el grado de informalidad y el tipo de interacción. Se encuestaron ocho OI nacionales, lo que significó una cobertura del 35\% de las identificadas.

De los datos se distinguen dos generaciones: en la primera, las OI buscaron recursos para fomentar la actividad innovadora y se basaron en extensores de modo informal o cuasi formal entre generadores de conocimiento y los usuarios, que conllevó, en algunos casos, a la dispersión de los recursos y resultados, o a un redimensionamiento de sus propósitos.

La segunda generación se caracteriza por el fomento explícito de la colaboración entre creadores de nuevo conocimiento y los usuarios, así como por la 
creación de entornos más propicios para la actividad innovadora y otros mecanismos que la promueven. Otra particularidad es que las OI de la primera generación han logrado, en esta etapa, una madurez institucional que les ha permitido conseguir propósitos definidos tanto en su gobernabilidad como en sus resultados concretos, y que los lazos de confianza refuerzan su labor de intermediación, sobre todo en su participación en el diseño de políticas públicas de fomento a la innovación (Pérez, 2011).

Para la identificación de los vacíos usando la figura 3, se consideraron los resultados de la encuesta sobre la frecuencia y grado de formalización. De este modo se identificaron tres vacíos estructurales: en patrocinio y negociación, en comercialización, financiamiento y explotación de los resultados, y en regulación y arbitraje (figura 4).

Figura 4. Identificación de vacíos estructurales en la actividad de innovación en México

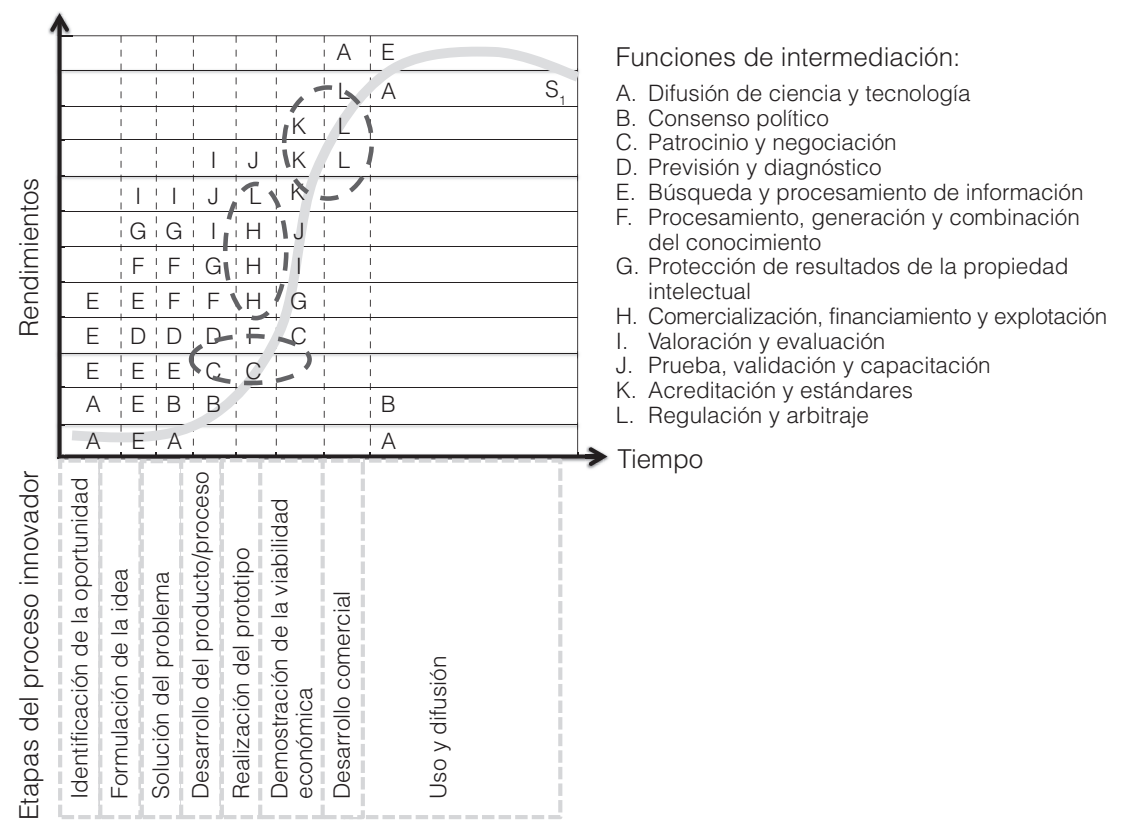

Fuente: Elaboración propia.

Estos resultados arrojan una situación inquietante, pues las que presentan vacíos estructurales están ligadas a la apropiación de los beneficios (comercialización y regulación) y a los incentivos para los actores (patrocinio y negocia- 
ción), lo que expresa la falta de eficacia y eficiencia de las OI en la mediación que realizan para la actividad innovadora.

En México, las funciones de apropiación y licenciamiento son incipientes, la Ley de Ciencia y Tecnología menciona recomendaciones en términos de regalías y creación de empresas, pero lo explicita solo para los centros públicos de investigación. No lo deja claro para institutos de investigación, IEs y otras dependencias de gobierno. Se crearon, por otra parte, mecanismos como las unidades de vinculación y de transferencia de conocimiento (UVTC u отс), sin embargo, estos no han logrado la eficacia en la comercialización de tecnología ni en su introducción al mercado (Pérez-Hernández \& CalderónMartínez, 2014).

De las conexiones entre las IEs y los CPI con las empresas, el flujo de información es el determinante principal. Donde hay bloqueos o apropiación negativa de conocimiento para otros fines, la circulación no fluye, no hay posibilidad de tareas de enlace, ni de profundizar en las actividades de promoción y comercialización de la actividad innovadora, justo los vacíos estructurales detectados.

$\mathrm{Al}$ considerar la evolución conjunta de las $\mathrm{OI}$ en México se puede aseverar que se encuentra en estadio inicial (DiMaggio \& Powell, 1983), pues no se ha logrado la estabilización de las estructuras, aunque existen algunas OI cuya presencia es más sólida y con reconocimiento y que se autoorganizaron a fin de responder a las exigencias del entorno, mas no como consecuencia de una mayor competencia entre sí.

\section{Reflexiones finales}

Crear los mecanismos para la conformación de entornos favorables para la innovación involucra la coordinación de esfuerzos para complementar la infraestructura y capacidades ya existentes. En países emergentes, la creación de esos entornos requiere de gran cantidad de recursos. La complejidad de los intercambios y las acciones productivas emprendidas territorialmente posibilitaron la creación de OI con flexibilidad organizativa, cuya capacidad orienta los apoyos públicos y privados hacia el fortalecimiento de un mercado de servicios tecnológicos y de aprendizaje de las pymes.

Respecto al papel de las OI en México puede afirmarse que su mayor relevancia consiste en dar confianza y certidumbre a las tareas de colaboración entre los actores de la actividad innovadora. En los análisis precedentes es evidente que ese "apoyo moral" es esencial para la transferencia de conocimiento y aprendizaje. Además, la función de enlace o mediador, como se comprobó en la investigación empírica, permite conciliar demandas, necesidades, recur- 
sos y capacidades disponibles, así como detectar los vacíos estructurales que obstaculicen la innovación.

En el caso mexicano, no obstante, algunas IES, CPI o gobiernos estatales, ante la necesidad de contar con intermediarios que faciliten la transferencia de conocimiento, han creado dependencias o estructuras que cumplan esa función. Esta situación generalmente se presenta en todos los países, sin embargo, en las naciones donde el marco institucional se encuentra en desarrollo y por la indefinición del papel de cada organización, las OI amplían sus funciones hasta ser multifuncionales. Esto conduce a la dispersión de esfuerzos, lo que origina numerosos vacíos estructurales en la intermediación que aquellas efectúan.

Respecto al comportamiento de las OI, según la evidencia expuesta en este artículo, se observa que su evolución sigue tres trayectorias. El primer grupo reúne a las que han madurado e incorporado las mejores prácticas de otras OI en el mundo por medio de la autoadaptación. Sin embargo, ante la escasa proliferación de otras OI que compitan por estos espacios, no ha habido un aprendizaje que les permita la evolución institucional.

En un segundo grupo se encuentran las OI de reciente creación que atienden espacios concretos, sobre todo a nivel regional o sectorial, donde la especialización de competencias las ha inducido a un cambio de funciones debido a las necesidades de sus clientes.

En un tercer grupo, se encuentran las OI que son una combinación de las dos primeras. Estas se relacionan con asociaciones empresariales o de productores que, ante la inercia institucional del espíritu corporativista de las empresas y cámaras empresariales mexicanas, han emprendido mecanismos alternos para enfrentar esos desafíos.

$\mathrm{Al}$ analizar en conjunto las $\mathrm{OI}$ mexicanas, en general se observa que sus estrategias son reactivas ante los cambios estructurales (económicos, productivos y tecnológicos) del país. Los esfuerzos han sido diversos: profesionistas y funcionarios con visión estratégica, oportunidad de negocio y disponibilidad de financiamiento público, entre otras situaciones, les ha permitido fungir en ese papel. Empero, los vacíos detectados hablan de elementos clave en el proceso de rentabilidad de la actividad innovadora, es decir, que las OI requieren de profesionalización y especialización, lo que no es fácil de construir en el corto plazo.

Finalmente, contribuir a solventar las debilidades del sistema de innovación llevaría a un diseño de políticas públicas ah doc a las necesidades del caso mexicano y, más aún, según la tecnología o sector, para consolidar los procesos de innovación. Pues el tipo de cualificación que se requiere para llenar esos vacíos no solo es necesario, sino crítico para consolidar la obtención de resultados e impactos que se esperan de la innovación. 


\section{Referencias}

Abernathy \& Utterback. (1978). Patterns of Industrial Innovation. Technology Review, 80(7), 40-47.

Baxter, Ch. \& Tyler, P. (2007). Facilitating Enterprising Places: The Role of Intermediaries in the United States and United Kingdom. En Polenska, K. (Ed.). The Economic Geography of Innovation (pp. 261-288). Cambridge Mass: Cambridge University Press.

Burt, R. (2002). The Social Capital of Structural Holes. En The New Economic Sociology: Developments in an Emerging Field (pp. 201-247). Nueva York: Russell Sage Foundation.

Casalet, M. (2012). Las relaciones de colaboración entre la universidad y los sectores productivos: una oportunidad para construir en la política de innovación. En Carrillo, J., Hualde, A. \& Villavicencio, D. (Coords.). Dilemas de la innovación en México (pp. 109-142). México: El Colegio de la Frontera Norte.

Casalet, M. (2008). Impacto de la sociedad del conocimiento en las estructuras institucionales y decisionales de los sistemas científicos: el caso de México. En Valenti, G. \& Casalet, M. (Coords.). Instituciones, sociedad del conocimiento y mundo del trabajo (pp.327-349). México: Flacso México/Plaza y Valdés.

Casalet, M. (2004). Los desafíos de la economía del conocimiento en el contexto institucional: de la jerarquía a la flexibilidad organizativa. Revista Mexicana de Sociología, 66(33), 119-142.

Casalet, M. (2000). The Institutional Matrix and its Main Functional Activities Supporting Innovation. En Cimoli, M. (Ed.). Developing Innovation Systems: Mexico in a Global Context (pp. 243-261). Londres: Continuum.

Casalet, M., Buenrostro, E. \& Becerril, G. (2008). La construcción de redes de innovación en los clusters de Software. Memorias del 3er Congreso Internacional de Sistemas de Innovacion para la Competitividad, Concyteg.

Casalet, M. \& González, L. (2006). El entorno institucional y la formalización de las redes en el sector electrónico de Chihuahua. En Villavicencio, D. (Coord.). La emergencia de dinámicas institucionales de apoyo a la industria maquiladora de México (pp. 49-87). México: Cámara de Diputados/uam/Miguel Ángel Porrúa.

Cimoli, M. (Comp.). (2000). Developing Innovation Systems: Mexico in a Global Context. Londres: Continuum.

DiMaggio, P. \& Powell, W. (1983). The Iron Cage Revisited: Institutional Isomorphism and Collective Rationality in Organizational Fields. American Sociological Review, 48, 147-160. 
Doner, R., Hicken, A. \& Ritchie, B. (2009). Political Challenges of Innovation in Developing World. Review of Policy Research, 26(1-2), 151-171.

Dutrenit, G. (Coord.). (2006). Diagnóstico de la política cientifica, tecnológica y de fomento a la Innovación en México (2000-2006). México: FСCyт/Conacyt.

Edquist, C. \& Johnson, B. (1997). Institutions and Organisations in Systems of Innovation. En Edquist, C. (Ed.). Systems of Innovation: Technologies, Institutions and Organizations. Londres y Washington: Pinter/Cassell Academic.

Ekboir, J., Dutrénit, G., Martínez, G., Torres, A. \& Vera-Cruz, A. O. (2009). Successful Organizational Learning in the Management of Agricultural Research and Innovation: The Mexican Produce Foundations. Research reports 162. International Food Policy Research Institute (IFPRI).

Freeman, Ch. (1987). Technology Policy and Economic Performance. Reino Unido: Pinter.

Galli, R. \& Teubal, M. (1997). Paradigmatic Shifts in National Innovation Systems. Systems of Innovation: Technologies, Institutions and Organizations (pp. 342-370). Londres: Pinter.

García, A., Lara, A. \& Taboada, E. (2004). La coordinación "híbrida” desde las perspectivas de Williamson y de Nooteboom. Análisis Económico, XIX(40), 101-117.

Howells, J. (2006). Intermediation and the Role of Intermediaries in Innovation. Research Policy, (35), 715-728.

Isiordia, P., Rodríguez, R. \& Sánchez, M. (2011). Organismos intermedios del aprendizaje empresarial y el desarrollo económico. En Villavicencio, D., Martínez, A. \& López, P. (Coords.). Dinámicas institucionales y políticas de innovación en México (pp. 43-56). México: uAm/Plaza y Valdés.

Jaso, M. (2011). El surgimiento de nuevos intermediarios para la innovación en México. En Villavicencio, D., Martínez, A. \& López, P. (Coords.). Dinámicas institucionales y politicas de innovación en México (pp. 19-42). México: UAm/Plaza y Valdés.

Lam, A. (2002). Modelos societales alternativos de aprendizaje e innovación en la economía del conocimiento. Revista Internacional de Ciencias Sociales, (171). Recuperado de http://www .oei.es/salactsi/lam.pdf

Lundvall, B., Johnson, B., Andersen, E. \& Dalum B. (2002). National Systems of Production, Innovation and Competence Building. Research Policy, 31, 213-231. 
Mantel, S. \& Rosegger, G. (1987). The Role of Third-Parties in the Diffusion of Innovations: A Survey. En Innovation: Adaptation and Growth (pp. 123-134). Amsterdam: Elsevier Science Publishers.

Nelson, R. (1998). The Co-evolution of Technology, Industrial Structure, and Supporting Institution. En Technology, Organization and competitiveness. Gran Bretaña: Oxford University Press.

Noteboom, B. (2000). Learning and Innovation in Organizations and Economies. Londres: Oxford University Press.

Pearson, A. (1990). Innovation Strategy. Technovation, 10(3), 185-192.

Pérez, P. (2014). Organizaciones intermedias en la actividad innovadora: el caso de México. México: Instituto Politécnico Nacional.

Pérez, P. (2011). Innovación y organismos intermedios en México: el caso de la biotecnología agrícola. En Villavicencio, D., Martínez, A. \& López, P. (Coords.). Dinámicas institucionales y politicas de innovación en México. México: Plaza y Valdés.

Pérez-Hernández, P. \& Calderón-Martínez, G. (2014). Análisis de los procesos de comercialización de tecnología en dos instituciones de educación superior mexicanas. Journal of Technology Managment \& Innovation, 9(3), 196-209.

Rodríguez, G. (2011). Actores de intermediación y enlace en la construcción de un sistema de innovación. El caso de la industria de software de Jalisco. En Villavicencio, D., Martínez, A. \& López, P. (Coords.). Dinámicas institucionales y políticas de innovación en México (pp. 81-111). México: UAm/Plaza y Valdés.

Rosenberg, N. (1994). Exploring the Black Box: Technology, Economics, and History. Cambridge: Cambridge University Press.

Shohert, S. \& Prevezer, M. (1996). UK Biotechnology: Institutional Linkages, Technology Transfer and the Role of Intermediaries. R\&D Management, 26, 283-298.

Stankiewicz, R. (1995). The Role of the Science and Technology Infrastructure in the Development and Diffusion of Industrial Automation in Sweden. En Technological Systems and Economic Performance: The Case of Factory Automation (pp. 165-210). Dordrecht: Kluwer Academic.

Stezano, F. (2012). Trasferencia de conocimientos entre ciencia e industria en el sector de biotecnología en México. En Carrillo, J., Hualde, A. \& Villavicencio, D. (Coord.). Dilemas de la innovación en México (pp. 143-184). México: El Colegio de la Frontera Norte. 
Stezano, F. (2009). Redes ciencia-industria para la transferencia en México, Estados Unidos y Canadá: Regimenes institucionales y tecnológicos y mecanismos de intermediación. Tesis de doctorado. México: Flacso México.

Szogs, A., Cummings, A. \& Chaminade, C. (2009). Building Systems of Innovation in Less Developed Countries: The Role of Intermediate Organizations, Sweden, CIRCLE, paper núm. 2009/01, University of Lund.

Tidd, J. \& Bessant, J. (2009). Managing Innovation: Integrating Technological, Market and Organizational Change. Inglaterra: John Wiley \& Sons.

Tirado, R. \& Luna, M. (2001). Las asociaciones empresariales y la construcción de redes de conocimiento. En La formación de redes de conocimiento. Una perspectiva regional desde México (pp. 119-160). Barcelona: IIs-UNAM/Anthropos.

Van der Meulen, B. (2007). Report for Prime Review 2007. En Workshop Intermediaries Organization and Process. Recuperado de www.prime-noe.org

Van Lente, H., Hekkert, M., Smits, R. \& Van Waveren, B. (2003). Roles of Systemic Intermediaries in Transition Processes. International Journal of Innovation Management, 7(3), 1-33.

Varela, G. (1997). Los patrones de vinculación universidad-empresa en Estados Unidos y Canadá y sus implicaciones para América Latina. En Casas Guerrero, R. \& Luna, M. (Coords.). Gobierno, Academia y Empresas en México (pp. 24-26). México: Plaza y Valdés Editores.

Vázquez, A. (2006). Surgimiento y transformación de clústers y milieus en los procesos de desarrollo. Revista Eure, 32(95), 72-92.

Vázquez, A. (2002). Endogenous Development: Networking, Innovation, Institutions and Cities. Londres: Routledge.

Whitman, Ch. (1989). Managing Technology Development: Perspectives of a Practitioner. Technovation, 9, 551-560.

Recibido el 26 de septiembre de 2014. Aceptado el 16 de junio de 2015. 